\title{
Enfermedad Tromboembólica Venosa en el período grávido puerperal. Diagnóstico, tratamiento y profilaxis.
}

\author{
Venous Thromboembolic Disease in the puerperal gravid period. \\ Diagnosis, treatment and prophylaxis. \\ Doença tromboembólica venosa no período gravídico puerperal. \\ Diagnóstico, tratamento e profilaxia.
}

Victoria Irigoín

ORCID: 0000-0002-6816-666 Hematóloga. Ex Asistente Cátedra de Hematología. Hospital de Clínicas.

UdelaR.

Internista.

Mariana Lorenzo ORCID: 0000-0002-6192-5053 Hematóloga. Asistente Cátedra de Hematología. Hospital de Clínicas. UdelaR

Sofía Grille

ORCID: 0000-0002-8099-2577 Hematóloga. Internista. Profeso Agregado Cátedra de Hematología

Hospital de Clínicas. UdelaR.

Valentín González ORCID: 0000-0002-0477-3794 Internista. Ex Asistente Clínica Médica B. Hospital de Clínicas. UdelaR.
Resumen: El embarazo y puerperio son situaciones asociadas fisiológicamente con un marcado aumento del riesgo de enfermedad tromboembólica venosa. El riesgo se estima entre 7 a 10 veces superior que en mujeres de igual edad y 15-35 veces superior que la población control, durante el puerperio y hasta la sexta semana post-parto. Los factores de riesgo son distintos durante el período prenatal y post-parto. Los métodos diagnósticos incluyen radiografía, angiotomografía de tórax y centellograma pulmonar ventilación-perfusión, ecodoppler venoso y venografía por resonancia nuclear magnética. El tratamiento supone un desafío que va desde la elección de la droga anticoagulante, los cambios en la farmacocinética durante el embarazo, entre otros. Se presentan algoritmos diagnósticos y terapéuticos.

Palabras clave: enfermedad tromboembólica venosa, diagnóstico, tratamiento, profilaxis.

Abstract: Pregnancy and the puerperium are physiologically associated with a markedly increased risk of venous thromboembolic disease. The risk is estimated between 7 to 10 times higher than in women of the same age and 15-35 times higher than the control population, during the puerperium and up to the sixth week postpartum. Risk factors are different during the prenatal and postpartum period. Diagnostic methods include radiography, chest angio-tomography and ventilation-perfusion lung scintigraphy, venous Doppler ultrasound, and magnetic resonance venography. Treatment involves a challenge that ranges from the choice of anticoagulant drug, changes in pharmacokinetics during pregnancy, among others. Diagnostic and therapeutic algorithms are presented.

Key words: venous thromboembolic disease, diagnosis, treatment, prophylaxis.

Resumo: A gravidez e o puerpério estão fisiologicamente associados a um risco acentuadamente aumentado de doença tromboembólica venosa. O risco é estimado entre 7 a 10 vezes maior do que em mulheres da mesma idade e 15 a 35 vezes maior do que na população controle, durante o puerpério e até a sexta semana pós-parto. Os fatores de risco são diferentes durante o período pré-natal e pós-parto. Os métodos de diagnóstico incluem radiografia, angiotomografia de tórax e cintilografia pulmonar de ventilação-perfusão, ultrassom Doppler venoso e venografia por ressonância magnética nuclear. $O$ tratamento envolve um desafio que vai desde a escolha do anticoagulante, mudanças na farmacocinética durante a gravidez, entre outros. Algoritmos diagnósticos e terapêuticos são apresentados.

Palavras-chave: doença tromboembólica venosa, diagnóstico, tratamento, profilaxia. 


\section{Introducción}

El embarazo y el puerperio son situaciones asociadas fisiológicamente con un marcado aumento del riesgo de Enfermedad Tromboembólica Venosa (TEV), con una prevalencia estimada de 1 en $1600^{1-4}$. La embolia pulmonar constituye la principal causa de muerte en países desarrollados probablemente en relación a una identificación subóptima de pacientes con riesgo aumentado que se beneficiarían de tromboprofilaxis; baja sospecha por la frecuente superposición de síntomas con los propios del embarazo y el temor del uso de ciertas técnicas diagnósticas por su eventual riesgo sobre el feto ${ }^{5}$.

Durante el período grávido puerperal se conjugan los 3 pilares de la tríada de Virchow: hipercoagulabilidad, estasis venosa y daño endotelial. Lo que explica que durante el embarazo el riesgo de TEV se estime entre 7 a 10 veces superior que en mujeres de igual edad y que sea 15-35 veces superior que la población control durante el puerperio y hasta la sexta semana post-parto ${ }^{6-9}$.

$80 \%$ de los eventos trombóticos en este período son venosos y $20 \%$ son arteriales. De los eventos trombóticos venosos, $80 \%$ de los casos se presentan a forma de Trombosis Venosa Profunda (TVP) y el $20 \%$ restante como embolia pulmonar (EP); de estos, el $50 \%$ ocurren durante el embarazo y el resto en el postparto.

Durante la gestación, las TVP son más frecuentemente proximales, masivas y en el miembro inferior izquierdo ${ }^{6,10}$. La trombosis de las venas pélvicas representa el $10-12 \%$ del total de las TVP en el embarazo y la trombosis en extremidades superiores el $2 \%$. Esta última forma de presentación se observa con mayor frecuencia en pacientes sometidas a técnicas de reproducción asistida. El riesgo de TEV durante el embarazo es similar en los 3 trimestres ${ }^{3,4,10}$.

Al estado de hipercoagulabilidad propio del embarazo en ocasiones se suman otros factores de riesgo que aumentan aún más la probabilidad de presentar esta complicación, siendo el más importante el antecedente de TEV previa. El riesgo relativo de diferentes factores de riesgo adicional surge de registros y estudios de caso control y se muestra en la Tabla $1^{8,11-13}$.

\begin{tabular}{|c|c|c|}
\hline & Factor de Riesgo & OR ajustado (95\% IC) \\
\hline \multirow{12}{*}{ TEV prenatal } & TEV previa & $24.8(17.1-36.0)$ \\
\hline & Enf médicas (LES, infección, anemia, venas varicosas) & $2.0-8.7(1.1-12.3)$ \\
\hline & Inmovilidad & $7.7(3.2-19.0)$ \\
\hline & Obesidad (IMC > 30) & $5.3(2.1-13.5)$ \\
\hline & Inmovilidad + Obesidad & 62 \\
\hline & Tabaquismo & $2.7(1.5-4.9)$ \\
\hline & Edad $>35$ & $1.3(1.0-1.7)$ \\
\hline & Paridad $>1$ & $1.5(1.1-1.9)$ \\
\hline & Preeclampsia & $3.1(1.8-5.3)$ \\
\hline & Preeclampsia con RCIU & $5.8(2.1-16.0)$ \\
\hline & Técnicas de reproducción asistida & $4.3(2.0-9.4)$ \\
\hline & Embarazo gemelar & $2.6(1.1-5.2)$ \\
\hline \multirow{4}{*}{$\begin{array}{l}\text { TEV } \\
\text { post-parto * }\end{array}$} & Hemorragia puerperal & $4.1(2.3-7.3)$ \\
\hline & Cesárea de urgencia & $3.6(3.0-4.3)$ \\
\hline & Infección post parto & $20.2(6.4-63.5)$ \\
\hline & Infección post cesárea & $6.2(2.4-16.2)$ \\
\hline
\end{tabular}

Las trombofilias ocupan un lugar relevante como factores de riesgo de TEV durante el período grávido-puerperal. Se ha reportado que aproximadamente $50 \%$ de las pacientes con esta complicación serán portadores de algún tipo de trombofilia; Hereditaria (TH) ○ Adquirida (TA) ${ }^{4,14-16}$. El riesgo de TEV durante el embarazo según tipo de trombofilia e incidencia global se muestran en la Tabla 2. 


\begin{tabular}{|l|l|l|}
\hline \multicolumn{2}{|c}{ Trombofilia } & \multicolumn{2}{c|}{ Prevalencia } \\
\hline Factor V ++Leiden Homocigota & $34.4(9.86-120.05)$ & $0.2-0.5 \%$ \\
\hline Factor V Leiden Heterocigota & $8.32(5.44-12.7)$ & $2.0-7.0 \%$ \\
\hline Factor II G20210A Homocigoto & $26.36(1.24-559.29)$ & Raro \\
& & \\
\hline Factor II G20210A Heterocigoto & $6.8(2.46-18.77)$ & $2.0 \%$ \\
\hline Déficit Proteína C & $4.76(2.15-10.57)$ & $0.2-0.3 \%$ \\
\hline Déficit Proteína S & $2.19(1.48-6)$ & $<0.1 \%$ \\
\hline Déficit Antitrombina & $4.76(2.15-10.57)$ & $<0.1 \%$ \\
\hline Ac Antifosfolipídicos & $15.8(10.9-22.8)$ & \\
\hline
\end{tabular}

\section{Diagnóstico}

Se requiere un alto nivel de sospecha y un adecuado conocimiento de los factores de riesgo asociados a la hipercoagulabilidad inherente al período grávido-puerperal para lograr un diagnóstico objetivo y precoz que reduzca las posibilidades de complicaciones en las mujeres embarazadas y puérperas.

Varios de los signos y síntomas de la TEV pueden confundirse con los derivados de ciertos cambios fisiológicos del embarazo como edema de miembros inferiores, taquicardia o disnea. Debe tenerse en cuenta que dado que las TVP pueden ser ilíacas, los síntomas pueden ser atípicos como dolor aislado a nivel inguinal, glúteo, flanco o abdomen. Cabe recordar que 30\% de los EP asocian TVP silente y el 40\% de TVP sintomáticas se acompañan de EP silente.

Es fundamental por tanto la identificación de parámetros que permitan establecer la probabilidad clínica de TEV, teniendo en cuenta que los scores utilizados frecuentemente a estos efectos no se encuentran validados en la población de mujeres embarazadas y puérperas.

En esta población, la herramienta utilizada con mayor frecuencia para la evaluación de la probabilidad clínica constituye la regla LEFt, surgida del resultado de la investigación de Chan WS y col y validada posteriormente por Le Moigne E y col ${ }^{17,18}$. Este score considera los siguientes factores como predictores de TVP: edema en pierna izquierda con $\geq 2 \mathrm{~cm}$ diferencia en la circunferencia de pantorrilla y presentación en el primer trimestre. El valor predictivo negativo (VPN) para TVP durante el embarazo es 100\% (95.8\% - 100\%) en ausencia de estas 3 variables. A pesar del alto VPN, este score necesita validación en un grupo mayor de pacientes ${ }^{17,18}$.

Con respecto a las técnicas diagnósticas, se describen a continuación riesgos y beneficios de las mismas durante el embarazo. En el puerperio se podrán aplicar las técnicas habituales en pacientes no embarazadas.

\section{Técnicas de confirmación diagnóstica de trombosis venosa profunda.}

\section{a) Ecodoppler venoso}

El ecodoppler del sistema venoso proximal se considera el test diagnóstico de primera línea para la confirmación de TVP durante el embarazo. Si el ecodoppler inicial demuestra anomalías en la compresibilidad y el flujo de las venas poplíteas y femorales compatibles con trombosis, se establece el diagnóstico de TVP y debe iniciarse tratamiento anticoagulante. Un resultado normal en una paciente con alta probabilidad clínica de TVP no debe excluir este diagnóstico dada la baja sensibilidad de esta técnica para la detección de TVP del sector venoso distal. En estos casos se recomienda la repetición de este estudio en los siguientes 3 y 7 días ${ }^{19-22}$. Cuando este estudio es persistentemente normal su VPN es elevado ${ }^{20}$.

\section{b) Venografía por Resonancia Nuclear Magnética}

En casos de sospecha de TVP del sector ilíaco debe considerarse como método diagnóstico de elección la venografía por resonacia nuclear magnética $(\mathrm{RM})$ o el ecodoppler venoso en manos de técnicos experimentados en el estudio de este sector anatómico ${ }^{19,20}$. La venografía por RM tiene una sensibilidad cercana al $100 \%$ para el diagnóstico de TVP pélvica en la población no embarazada, no existiendo datos certeros sobre la misma en este grupo particular de pacientes. 
De acuerdo a un gran estudio de cohorte de Ontario, Canadá ${ }^{23}$ la exposición a la RM en el primer trimestre no se asoció a un riesgo aumentado de daño para el feto o parto pretérmino.

Sin embargo, el uso de Gadolinium como contraste se desaconseja porque podría producir riesgo aumentado de enfermedades reumatológicas inflamatorias, infiltración cutánea, parto pretérmino y muerte neonatal. Por lo tanto, de ser necesario, la Sociedad Americana de Ginecoobstetricia (ACOG) recomienda la revisión caso a caso evaluando el riesgo-beneficio ${ }^{24}$.

\section{c) Angio - Tomografía Computada}

En casos de sospecha de TVP en el sector ilíaco y de no contar con RM, puede evaluarse el uso de Angio Tomografía Computada (angio-TC). Sus principales características se describen en el párrafo de embolia pulmonar.

\section{Técnicas de Confirmación Diagnóstica de Embolia Pulmonar}

Clásicamente se ha percibido como una preocupación el uso de técnicas diagnósticas de imagen durante el embarazo por el temor a los potenciales efectos adversos sobre el feto de la irradiación y /o el contraste.

En ocasiones, estos motivos se citan como argumentos para evitar un uso adecuado de estas técnicas, lo que conlleva un riesgo inaceptable de sub-diagnóstico de una patología potencialmente mortal como la EP. Cabe destacar que no existen escores validados para establecer la probabilidad clínica de EP en la población de mujeres embarazadas, y que los estudios no imagenológicos como el electrocardiograma y gasometría arterial son de limitado valor a efectos diagnósticos.

La dosis mínima asociada con riesgo de teratogenicidad en seres humanos no está claramente establecida. Sobre la base de estudios animales se considera que como valor de corte práctico, la dosis de 0,1 Gy. Dosis mayores pueden asociarse con posibles anomalías fetales ${ }^{25-27}$. Las técnicas de imagen más frecuentemente utilizadas a estos efectos no se asocian con niveles elevados de exposición fetal a la irradiación por lo que no debe retrasarse su solicitud frente a la sospecha de esta entidad (Tabla 3).

Tabla 3: Dosis de irradiación fetal estimada de las diferentes técnicas de imagen 28-30

\begin{tabular}{|l|l|}
\multicolumn{1}{|c|}{ Técnica } & \multicolumn{1}{c|}{ Radiación (Gy) } \\
\hline Rx Tx & 0,000001 \\
\hline Centellograma Ventilación & $0,00028-0,00051$ \\
\hline Centellograma perfusión (mitad de dosis) & $0,00014-0,00025$ \\
\hline Angio TAC & $0,000003-0,000131$ \\
\hline
\end{tabular}

\section{a) Radiografía de Tórax}

En pacientes con sospecha de EP que se encuentran hemodinámicamente estables, la radiografía de tórax $(\mathrm{Rx} T \mathrm{x})$ permite descartar diagnósticos diferenciales tales como neumopatía aguda o neumotórax. Las mujeres embarazadas, por su edad, en general tienen baja tasa de enfermedad pulmonar y la RxTx será normal en más del $50 \%$ de los casos. La RxTx puede evidenciar hallazgos no específicos de EP como atelectasia, derrame pleural, opacidad focal, oligohemia o edema pulmonar pero su sensibilidad y especificidad son bajas por lo que su principal utilidad es la valoración de diagnósticos alternativos ${ }^{25}$.

\section{b) Angio-TC de Tórax}

Constituye el método diagnóstico de elección en pacientes con RxTx anormal ${ }^{19}$. Esta técnica conlleva menor exposición del feto a la irradiación que el centellograma ventilación/perfusión $(\mathrm{V} / \mathrm{Q})$ durante los 3 trimestres de la gestación ${ }^{19}$. Sin embargo, este estudio expone a la madre a una considerable dosis de irradiación que puede alcanzar 20 mGy en tórax y en tejido mamario, el cual durante el embarazo se encuentra en una situación particularmente sensible al efecto de la irradiación. Esto se asocia con un riesgo acumulado incrementado de cáncer de mama ${ }^{31}$. La dosis de irradiación sobre este tejido puede reducirse hasta $50 \%$ con el uso de protectores mamarios ${ }^{31}$.

La angio-TC tiene mayor sensibilidad que el centellograma V/Q para el diagnóstico de EP no masiva y masiva, y permite descartar diagnósticos diferenciales. Este estudio presenta menor sensibilidad que en pacientes no embarazadas dado el estado hiperdinámico asociado a la 
gestación, siendo su VPN cercano a 98\%. Generalmente es una técnica de rápido acceso en la mayoría de los centros de atención terciaria lo que lleva a su uso extendido para el diagnóstico de EP.

\section{c) Centellograma pulmonar de ventilación-perfusión (V/Q)}

Método útil en el diagnóstico de EP en pacientes sin alteraciones pulmonares previas. Se asocia con menor exposición a la irradiación del tejido mamario materno, pero con exposición algo mayor sobre el feto. En pacientes con RxTx normal en ocasiones puede omitirse la fase de ventilación, reduciendo así la dosis de irradiación fetal ${ }^{19}$.

No constituye la técnica de elección en casos de EP grave, y debe tenerse en cuenta que no en todos los centros se cuenta con fácil y rápido acceso a esta técnica diagnóstica. De encontrarse disponible puede considerarse como la mejor opción diagnóstica dada la menor exposición materna a la irradiación.

En ocasiones y teniendo en cuenta la asociación entre EP y TVP asintomática puede optarse por la realización de un ecodoppler venoso de MMII, que de confirmar la presencia de TVP justifica el inicio de terapia anticoagulante y podría evitar la necesidad de realización de técnicas de imagen ya que ante el diagnóstico de TVP la conducta terapéutica es igual.

En la figura 1 y 2 se muestran los algoritmos diagnósticos propuestos en caso de TVP y/o EP.

Sospecha de TVP

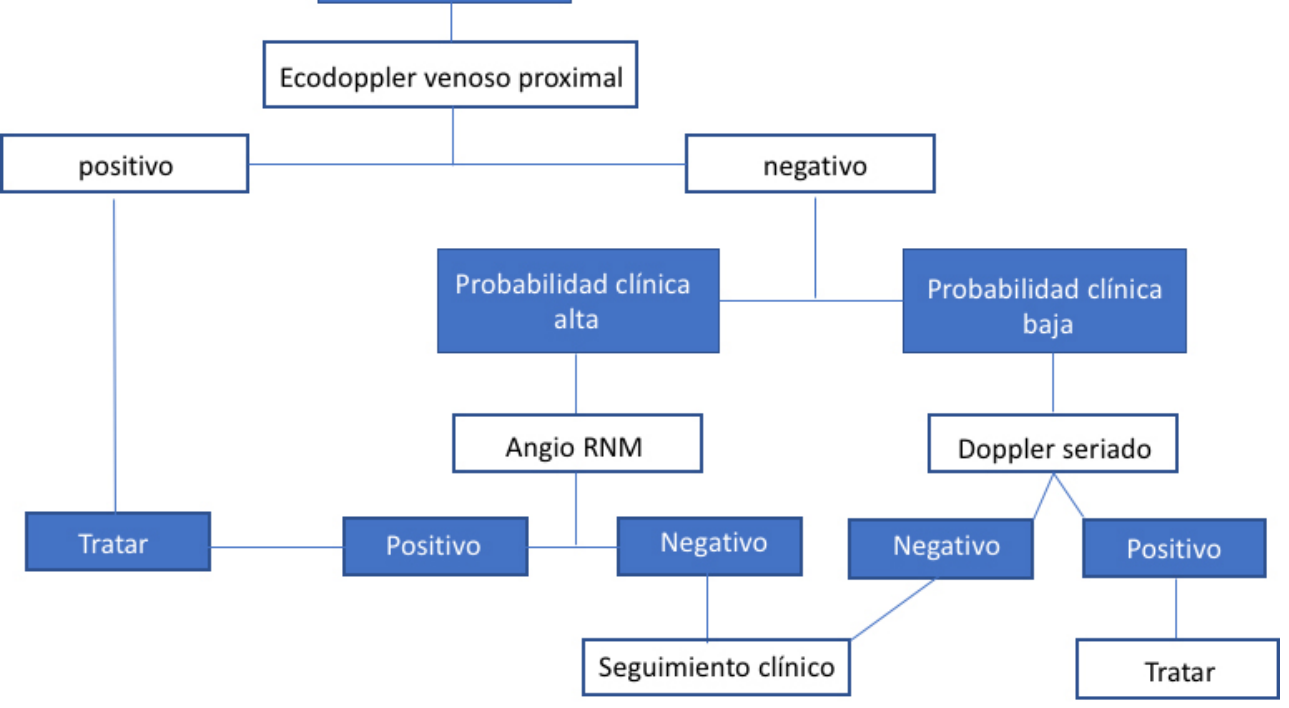




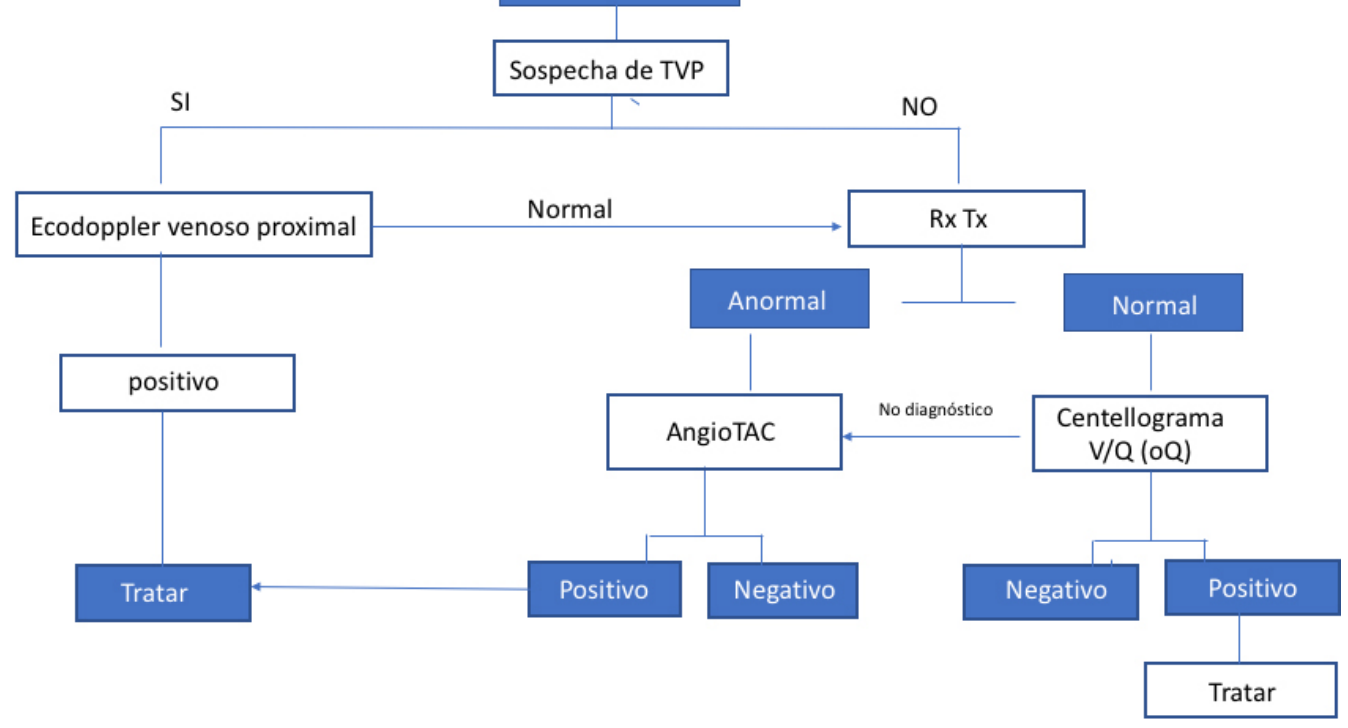

\section{Tratamiento}

El tratamiento de la TEV en el embarazo supone un desafío por diversas dificultades: la escasa evidencia de buena calidad, la elección de la droga anticoagulante, los cambios en la farmacocinética a lo largo del embarazo, los aspectos vinculados a la seguridad y limitación de la anestesia neuroaxial, entre otras. Para la toma de decisiones, ante un evento tromboembólico se debe valorar: el tipo de evento, el momento del embarazo y el riesgo de sangrado.

\section{Anticoagulación farmacológica}

\section{a) ¿Con qué fármaco?}

La warfarina atraviesa la placenta, es teratogénica y provoca anticoagulación fetal y eventos de hemorragia cerebral, por lo cual no debe utilizarse en el embarazo (excepto situaciones particulares), y especialmente en el primer trimestre.

Las heparinas no atraviesan la placenta ni se excretan en la leche materna, sin embargo tampoco contamos con evidencia de calidad para definir seguridad y eficacia.

En este sentido, se ha publicado una revisión sistemática de Cochrane en el 2014, que tuvo por objetivo comparar eficacia entre Heparina de bajo peso molecular (HBPM), heparina no fraccionada (HNF) y warfarina en TVP en el embarazo, donde se concluyó que no se disponían de estudios controlados randomizados ${ }^{32}$. Por ello, el uso de heparinas se sustenta en extrapolaciones de estudios en no embarazadas. No hay estudios randomizados que comparen la eficacia y seguridad de la HNF versus HBPM en el embarazo, pero sí existe vasta evidencia observacional y experiencia clínica que soporta el uso de HBPM.

Una revisión sistemática y meta-análisis publicada en el 2005, mostró que la HBPM es segura y eficaz para el tratamiento y profilaxis en el embarazo ${ }^{33}$. Se incluyeron 2777 embarazos en los que se utilizaron diversas HBPM. No se identificaron muertes maternas y $95 \%$ fueron nacidos vivos. En el subgrupo de tratamiento hubo baja recurrencia TEV (1.15\%), baja frecuencia de sangrado significativo (1.72\% y mayormente por causa obstétrica), ningún caso de trombocitopenia inducida por heparina (HIT), ni de fracturas osteoporóticas. Estos datos muestran un mejor perfil de seguridad al reportado con el uso de HNF.

Por tanto, las drogas de elección son las HBPM, y es lo recomendado por las diversas guías (recomendación 1B) ${ }^{20,34}$. En nuestro país están disponibles nadroparina y enoxaparina.

Las guías refieren que podría utilizarse HNF de preferencia en situación de alto riesgo de sangrado como la instalación del evento tromboembólico en el periparto, así como en la embolia pulmonar masiva.

Las alternativas en caso de alergia a HBPM son: danaparoide, argatroban, fondaparinux. Tienen diversos perfiles de seguridad pero han sido utilizados con buenos resultados ${ }^{35}$. 
No hay evidencia suficiente de seguridad y eficacia de los anticoagulantes orales directos, por lo que no están recomendados en el embarazo.

\section{b) ¿A qué dosis?}

Nuevamente, la evidencia se extrapola de ensayos en pacientes no embarazadas. Es conocido el hecho de que existen cambios farmacocinéticos y del filtrado glomerular a lo largo del embarazo, y está demostrado que la vida media de la enoxaparina aumenta con el curso del embarazo ${ }^{36}$.

No está claro si la dosis terapéutica debe ser administrada una o dos veces al día (ej, enoxaparina $1.5 \mathrm{mg} / \mathrm{kg} /$ día o $1 \mathrm{mg} / \mathrm{kg} \mathrm{c} / 12$ horas). Evidencia observacional del manejo clínico en la vida real muestra que la mayoría de las pacientes son tratadas con una dosis al día 37. Las guías RCOG no se definen por una pauta en particular. Sí está estandarizado que el peso a utilizar para el cálculo de dosis es el pre-gestacional o el de la consulta precoz de embarazo (tabla 4). En nuestro medio, debido a la disponibilidad restringida de las presentaciones de HBPM, habitualmente se realiza el esquema dos veces al día.

\begin{tabular}{|l|l|c|}
$\begin{array}{c}\text { Peso pre-estacional o de consulta } \\
\text { gestacional precoz }\end{array}$ & Dosis de enoxaparina (s.c) & \multicolumn{1}{|c|}{ Dosis de nadroparina (s.c) } \\
\hline$<50 \mathrm{~kg}$ & $40 \mathrm{mg} \mathrm{c} / 12 \mathrm{hr} 060 \mathrm{mg} / \mathrm{d}$ & $0.4 \mathrm{cc} \mathrm{c/12hr}$ (3800Ul) 0 0.6cc/día (5700 Ul) \\
\hline $50-69 \mathrm{~kg}$ & $60 \mathrm{mg} \mathrm{c} / 12 \mathrm{hr} 090 \mathrm{mg} / \mathrm{d}$ & \\
\hline $70-89 \mathrm{~kg}$ & $80 \mathrm{mg} \mathrm{c} / 12 \mathrm{hr} 0120 \mathrm{mg} / \mathrm{d}$ & \\
\hline $90-109 \mathrm{~kg}$ & $100 \mathrm{mg} \mathrm{c} / 12 \mathrm{hr} 0150 \mathrm{mg} / \mathrm{d}$ & \\
\hline $110-125 \mathrm{~kg}$ & $120 \mathrm{mg} \mathrm{c} / 12 \mathrm{hr} 0180 \mathrm{mg} / \mathrm{d}$ & \\
\hline$>125 \mathrm{~kg}$ & Valorar & \\
\hline
\end{tabular}

\section{c) ¿Se debe monitorizar con anti-Xa?}

Los mencionados cambios farmacocinéticos han avalado en el pasado el uso de la actividad anti-Xa como parámetro de control de la anticoagulación con HBPM. El esquema de $1 \mathrm{mg} /$ $\mathrm{kg}$ cada $12 \mathrm{hr}$ logra medidas estables de anti-Xa. Sin embargo, la amplia variabilidad según el curso del embarazo, según el tipo de ensayo, así como variabilidad inter-laboratorio, sumado a la vasta evidencia observacional del uso seguro de HBPM, hacen desaconsejar el monitoreo sistemático. Las excepciones en las que se recomienda su determinación (pico esperado de $0.5-1.2 \mathrm{U} / \mathrm{ml}$ a las 3 horas) son: peso menos de $50 \mathrm{~kg}$ o más de $90 \mathrm{~kg}$, insuficiencia renal, recurrencia de TEV bajo anticoagulación ${ }^{38-40}$ o situaciones de alto riesgo tromboembólico (ej: válvulas mecánicas).

\section{d) ¿Hasta cuándo se mantiene la anticoagulación y con qué droga?}

El riesgo tromboembólico persiste durante todo el embarazo y hasta 6 semanas luego del parto, lo que expone a la paciente a un inaceptable riesgo de recurrencia ${ }^{41}$. Esto justifica la persistencia del tratamiento durante todo el embarazo y hasta 6 semanas post parto, debiéndose cumplir al menos 3 meses de tratamiento a dosis plenas.

La dosis a la cual se debe continuar en tratamiento, no está aclarada. Evidencia extrapolada de pacientes no embarazadas demuestran que las dosis intermedias o de profilaxis no son adecuadas por la alta tasa de recurrencia. En el embarazo, no existen estudios randomizados comparativos. Una revisión sistemática de estudios en que se utilizaron dosis intermedias, reportan 1 recurrencia en 156 pacientes, sin embargo, los propios autores refieren probable sobreestimación de la efectividad por escasos casos de EP e inclusión de casos de TVP distal ${ }^{41}$. Existen otras revisiones que reportan baja tasa de recurrencia con dosis intermedias ${ }^{33,42}$. Las guías RCOG sugieren mantener la dosis de anticoagulación por todo el período, dado ausencia de evidencia contundente y buen perfil de seguridad ${ }^{34}$.

Al finalizar el puerperio (y una vez completado los 3 meses de tratamiento), se debe evaluar riesgo individual de recurrencia y definir duración de anticoagulación.

La warfarina puede ser dada de manera segura en la lactancia, por lo que es una opción válida, una vez superado el riesgo hemorrágico. 


\section{Manejo de anticoagulación periparto}

Dado que la finalización del parto tiene alto riesgo de sangrado, se considera la suspensión momentánea de la anticoagulación. Resulta evidente que la mejor conducta serála individualizada, según el balance entre riesgo de recurrencia y riesgo de sangrado.

Se la debe instruir a la paciente de no administrarse la dosis de HBPM en caso de estar empezando el trabajo de parto. En caso de fin de embarazo no programado y que se haya administrado la inyección, es muy bajo el riesgo de sangrado.

Si la TEV se presenta en el período periparto (alto riesgo tromboembólico de suspender la HBPM), el uso de HNF como puente dado su corta vida media puede ser útil. Se inicia con bolo de $80 \mathrm{U} / \mathrm{kg}$, siguiendo con infusión de $18 \mathrm{U} / \mathrm{kg} / \mathrm{hr}$ para lograr un aptt 1.5 a 2.5 veces superior al normal. Se chequea a las 6 horas de iniciado el bolo y luego cada 4-6 horas hasta lograr rango. Una vez en rango, se puede testar una vez al día.

\section{¿Cuándo usar un filtro cava?}

Al igual que en pacientes fuera del embarazo, la colocación de filtros cava están indicados en caso de contraindicación para la anticoagulación o EP recurrente a pesar de correcta anticoagulación.

Puede considerarse el uso de filtro cava en la instalación de una TVP ilíaca periparto para reducir el riesgo de EP.

En todos los casos la colocación de filtros removibles es lo recomendado ${ }^{43,44}$, y retirarlo luego de finalizado el puerperio y sin contraindicación para la anticoagulación.

\section{Profilaxis}

La utilización de tromboprofilaxis farmacológica durante el embarazo y puerperio es un tema de gran controversia. No hay ensayos clínicos randomizados que evalúan la tromboprofilaxis en el embarazo o puerperio dado que los mismos han sido difíciles de implementar ${ }^{45}$. La mayoría de las recomendaciones se basan en estudios retrospectivos y en la opinión de expertos y por tanto tienen un nivel de evidencia bajo. Las recomendaciones por las guías clínicas internacionales a menudo varían en cuanto a algunas indicaciones de profilaxis. A pesar de esto, la mayoría de los expertos coinciden que es un escenario de elevado riesgo y que a las pacientes de riesgo alto/ intermedio se les debería ofrecer tromboprofilaxis farmacológica.

El riesgo de TEV debe ser evaluado en cada paciente de forma individualizada teniendo en cuenta los diferentes factores de riesgos trombóticos que han sido mencionados previamente. Esta evaluación del riesgo es dinámica, por lo que debe realizarse en distintos momentos durante el embarazo y puerperio. También es necesario valorar el riesgo-beneficio del tratamiento anticoagulante teniendo en cuenta el efecto sobre la madre y el feto y elegir cuál es el fármaco, la pauta de administración y la dosis más adecuada.

La tromboprofilaxis puede ser farmacológica o mecánica. Las estrategias de profilaxis mecánica incluyen la deambulación precoz, medias de compresión venosa graduada y compresión neumática intermitente. Si bien los métodos de compresión neumática intermitente han demostrado prevenir el desarrollo de TEV en pacientes quirúrgicos, hay escasa evidencia de su eficacia en la mujer embarazada y/o puerpera46. El American Congress of Obstetricians and Gynecologists (ACOG) recomienda la utilización de compresión neumática intermitente en todas las pacientes a las que se les realice cesárea y que ya no estén recibiendo tromboprofilaxis farmacológica ${ }^{47}$.

La tromboprofilaxis farmacológica se puede realizar con HNF o HBPM ya que no cruzan la placenta y son seguras en el embarazo. Las HBPM son los anticoagulantes de elección para realizar profilaxis farmacológica por largo período en el embarazo debido a su buen perfil de seguridad. La HNF es una alternativa válida, pero tiene mayor riesgo de inducción de HIT y osteoporosis con su uso prolongado. Las ventajas de la HNF es su bajo costo y que tiene una vida media corta lo cual puede ser útil para el manejo en el periparto en pacientes de alto riesgo trombótico ${ }^{48}$.

Los estudios para estimar el riesgo de hemorragia en mujeres embarazadas bajo tromboprofilaxis farmacológica son limitados. La proporción de TEV fatal varía desde $0 \%$ a $1.91 \%$ con un promedio de $0.68 \%$ (IC $95 \% 0.41 \%-0.96 \%$ ), sin embargo no hay datos suficientes para estimar la tasa de sangrado fatal vinculado directamente a $\mathrm{HBPM}^{49}$. En un 
meta-análisis que combina los datos de 8 ensayos clínicos randomizados para evaluar el rol de la HBPM a dosis profilácticas en prevenir las complicaciones mediadas por la placenta, el riesgo de sangrado mayor durante el embarazo fue de $0.2 \%$ ( 1 de 470 ) y el riesgo de sangrado mayor en el post-parto fue de $0.6 \%$ (3 de 473)50. Otro meta-análisis publicado por Greer IA et al. evalúo la seguridad de la HBPM en 2777 embarazos y observó que el sangrado mayor durante la gestación fue de $0.43 \%$ (95\% IC, 0.22\% - 0.75\%) y en el puerperio de $0.94 \%$ (95\% IC, 0.61\% $-1.37 \%)^{51}$.

Nuestra experiencia en el Hospital de Clínicas aplicando las guías de tromboprofilaxis del Royal College of Obstetricians and Gynaecologists (RCOG) 2009 fue de 1 episodio de TEV en 1035 pacientes sin haber registrado eventos hemorrágicos mayores, por lo que hemos observado buen perfil de seguridad con la utilización de HBPM a dosis profilácticas ${ }^{52}$.

Los episodios de hemorragia en Estados Unidos son una importante causa de mortalidad maternal y entre 2011 y 2013 corresponden al $11.4 \%$ de las causas de muerte materna. Por lo tanto, basándose en datos del riesgo hemorrágico con HBPM de la población no materna, algunos autores asumen que el riesgo de sangrado fatal con profilaxis con HBPM es 2 a 3 veces más común que los episodios de TEV durante el embarazo, por lo que la profilaxis con HBPM tendría que evitar al menos 2 a 3 eventos de TEV más por cada sangrado mayor para proporcionar un beneficio neto ${ }^{53,54}$.

En base a estos y otros datos es que algunos expertos consideran que es probable que no haya un beneficio clínico neto para realizar tromboprofilaxis con HBPM cuando el riesgo absoluto de TEV durante el embarazo o puerperio es menor a $1 \%$ y es muy probable que exista beneficio clínico neto cuando el riesgo absoluto de TEV es mayor a 3\%. Entre 1 y $3 \%$ la indicación es aún más discutida ${ }^{54}$.

Por todo lo anterior es que, no está recomendada la tromboprofilaxis de rutina en todas las pacientes embarazadas y/o puérperas, y por el contrario, se debe realizar una evaluación cuidadosa de todos los factores de riesgo trombóticos y del riesgo hemorrágico. Para facilitar esta decisión clínica, diferentes sociedades científicas internacionales han publicado guías clínicas haciendo una estimación del riesgo trombótico y guiando la tromboprofilaxis. Dentro de ellas están las guías del The American College of Chest Physicians (ACCP), Royal College of Obstetricians and Gynaecologists (RCOG), American College of Obstetricians and Gynecologists (ACOG), French College of Gynaecologists and Obstetricians (CNGOF), Society of Obstetricians and Gynaecologists of Canada, Sociedad de Obstetricia y Ginecología de Buenos Aires (SOGIBA), entre otras ${ }^{20,47,55-58}$. Dada la ausencia de evidencia clínica de calidad es que existen diferencias entre las distintas guías.

En estas guías la tromboprofilaxis está recomendada en mujeres con un riesgo de TEV estimado mayor a 5\% y no se recomienda realización de tromboprofilaxis si es riesgo es inferior a $1 \%$. La realización de tromboprofilaxis con un riesgo de TEV estimado entre 1 a $5 \%$ es controversial ${ }^{55,58}$

Las guías del RCOG usan un sistema de score para determinar el riesgo (bajo, intermedio y alto) y guiar la tromboprofilaxis ${ }^{55}$.

Nuestra experiencia con la aplicación de las guías del RCOG 2009 ha sido buena. Entre enero de 2014 y Diciembre de 2016 hemos registrado 1035 pacientes embarazadas y puérperas a las que se les ha aplicado el sistema de riesgos de la RCOG 2009 y hemos evaluado que en nuestra población el 3.0\% corresponden a pacientes de alto riesgo y $35.4 \%$ de riesgo intermedio, por lo que $38.4 \%$ de las pacientes de nuestro servicio tenían indicación de recibir tromboprofilaxis farmacológica. La población de pacientes del Hospital de Clínicas tiene una elevada frecuencia de pacientes de alto riesgo obstétrico. Es de destacar que no tuvimos episodios hemorrágicos mayores ${ }^{52}$.

Las guías de ACOG usan una estrategia de screening basado en la historia familiar y personal de TEV, la presencia de trombofilia y otros factores de riesgo ${ }^{49}$. Las recomendaciones de la ACCP categoriza los factores de riesgo en mayores y menores y se sugiere tromboprofilaxis si tiene un factor de riesgo mayor o al menos 2 menores ${ }^{20}$.

Basado en nuestra experiencia previa con las guías del RCOG 2009 es que nosotros preferimos guiar nuestras recomendaciones basándonos en las guías del RCOG $2015^{55}$. En las mismas se recomienda realizar una evaluación del riesgo trombótico en el primer control del embarazo, frente a los ingresos, en el postparto inmediato y frente a cualquier cambio o patología intercurrente durante la evolución de la paciente (figura 3 y 4). 
TEV previa (excepto TEV provocada)

-Ingreso hospitalario por patología médica o quirúrgica

-TEV provocada

-Anticuerpos antifosfolípidos positivos

(AAF) o Trombofilia hereditaria de alto

riesgo* asintomática (sin ETEV previa)

-Comorbilidades médicas (ej: cáncer,

insuficiencia cardíaca, enfermedad

autoinmune en actividad, síndrome

nefrótico, diabetes mellitus tipo 1 con

nefropatía, anemia drepanocítica, adicto a

drogas intravenosas)

-Procedimientos quirúrgicos durante

embarazo

-Síndrome de hiperestimulación ovárica en el primer trimestre

\section{-Edad $>35$ años}

-Obesidad (IMC > 30kg/m2)

-Paridad $\geq 3$

-Fumadora

-Gruesas venas varicosas

-Inmovilidad (ej paraplejia)

-Pre-eclampsia actual

-Historia familiar de TEV no provocada o asociada a estrógenos en familiar de 1er

grado menor de 50 años

-Trombofilia Hereditaria asintomática de

bajo riesgo*

-Embarazo múltiple o terapia

reproducción asistida o fertilización in

vitro.

-Factores de riesgo transitorios: Infección

sistémica actual, viaje mayor $4 \mathrm{hs}$,

deshidratación, hiperémesis.

Trombofilia Hereditaria de alto riesgo: deficiencia de AT, S y C, Factor V Leiden homocigoto, Factor II G20210A homocigoto, doble heterocigoto.

Trombofilia de Bajo riesgo: Factor $\mathrm{V}$ Leiden heterocigoto, Factor II G20210A heterocigoto
TEV: tromboembolia venosa

AAF: anticuerpos antifosfolipidos títulos moderados o altos.
ALTO RIESGO

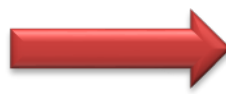

Requiere tromboprofilaxis

con HBPM durante todo

embarazo

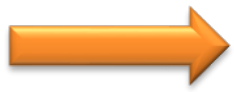

\section{RIESGO INTERMEDIO}

Se recomienda

tromboprofilaxis con HBPM

$\geq 4$ factores

Se recomienda tromboprofilaxis

con HBPM desde 1er trimestre

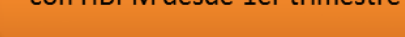

3 factores

Se recomienda tromboprofilaxis con HBPM desde semana 28.

\section{BAJO RIESGO}

$<3$ factores

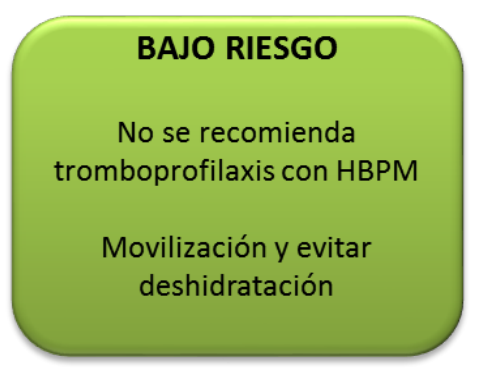

Adaptado de Royal College of Obstetrician and Gynaecologists Guidelines 


-TEV previa
-Requerimiento de HBPM durante
embarazo
-Anticuerpos antifosfolípidos positivos
(AAF) o Trombofilia hereditaria de alto
riesgo* asintomática (sin ETEV previa)
-Trombofilia Hereditaria asintomática de
bajo riesgo* + Historia familiar de TEV no
provocada o asociada a estrógenos en
familiar de 1er grado menor de 50 años

-Cesárea de emergencia

-Comorbilidades médicas (ej: cáncer, insuficiencia cardíaca, enfermedad autoinmune en actividad, síndrome nefrótico, diabetes mellitus tipo 1 con nefropatía, anemia drepanocítica, adicto a drogas intravenosas)

-Estadía hospitalaria prolongada ( $\geq 3$ días) en puerperio

- Procedimiento quirúrgico distinto a reparación del periné.

-Obesidad (IMC $>40 \mathrm{~kg} / \mathrm{m} 2$ )

-Edad $>35$ años
-Obesidad (IMC $>30 \mathrm{~kg} / \mathrm{m} 2)$
-Paridad $\geq 3$
-Fumadora
-Gruesas venas varicosas
-Infección sistémica actual
-Inmovilidad
-Viaje mayor $4 \mathrm{hs}$
-Pre-eclampsia actual
-Cesárea de coordinación
-Historia familiar de TEV no provocada o
asociada a estrógenos en familiar de $1 \mathrm{er}$
grado menor de 50 años
-Trombofilia Hereditaria de bajo riesgo
asintomática
- Parto pretérmino (<37 semanas) o óbito
-Trabajo de parto prolongado (>24hs)
-Embarazo múltiple
- Hemorragia postparto mayor 1 It o que
requiere transfusión
-maniobras de rotación intrauterinas
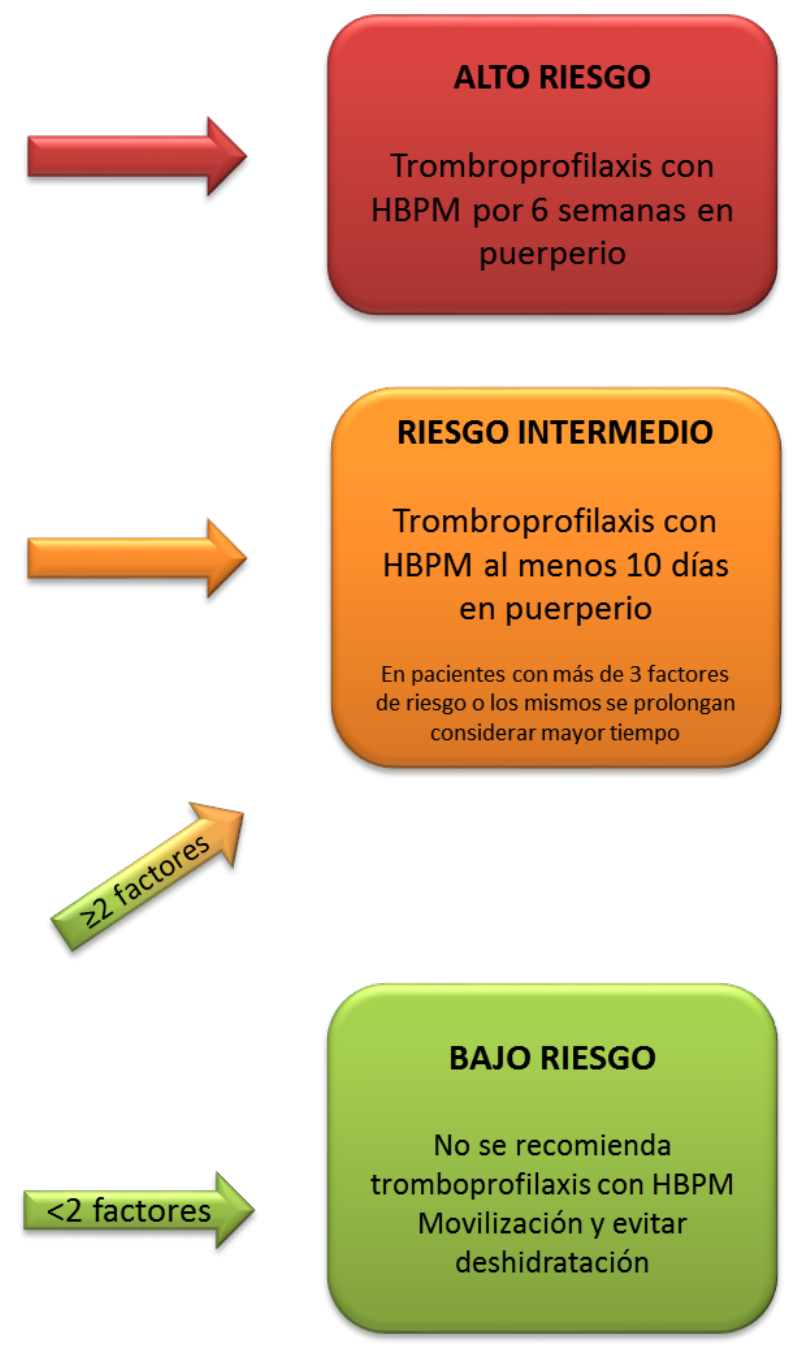

* Trombofilia Hereditaria de alto riesgo: deficiencia de AT, S y C F Factor $V$ Leiden homocigoto, Factor II G20210A homocigoto, doble heterocigoto.

Trombofilia de Bajo riesgo: Factor $V$ Leiden heterocigoto, Factor II G20210A heterocigoto

TEV: tromboembolia venosa

AAF: anticuerpos antifosfolípidos títulos moderados o altos

Adaptado de Royal College of Obstetrician and Gynaecologists Guidelines

Este riesgo se clasificará en alto, intermedio y bajo con lo cual se decide la indicación de tromboprofialxis y la duración de la misma. Las guías del RCOG 2015 han realizado algunas modificaciones con respecto a las guías previas del 2009. Dentro de las más destacadas está el hecho de que durante el embarazo toda paciente que requiera ingreso hospitalario por patología obstétrica, médica o quirúrgica debe considerarse de riesgo intermedio. Asimismo, durante el embarazo dependiendo de cuantos factores de riesgo menores presente la paciente es si la tromboprofilaxis se realiza durante todo el embarazo o solo luego de la semana 28 de gestación. Además, a las pacientes de alto riesgo se sugiere tromboprofilaxis durante todo embarazo y hasta 6 semanas en el puerperio y a las de riesgo intermedio durante embarazo y 10 días en el puerperio ${ }^{55}$.

La evaluación del riesgo trombótico debe ser siempre acompañada de una cuidadosa evaluación del riesgo hemorrágico y se desaconseja la realización de tromboprofilaxis frente a las siguientes situaciones: enfermedad hemorrágica conocida (ej.: enfermedad de Von Willebrand, hemofilia u otra coagulopatía), sangrado activo, mujeres en las que se considera alto riesgo hemorrágico (ej.: placenta previa con sangrado), trombocitopenia menor a $50.000 / \mu \mathrm{L}$, stroke isquémico o hemorrágico en las 4 semanas previas, enfermedad renal crónica con clearence de creatinina menor a $30 \mathrm{ml} / \mathrm{min}$ ), enfermedad hepática severa con várices esofágicas conocidas o 
alteración del tiempo de protrombina, hipertensión arterial no controlada (mayor a 200mmHg de sistólica y $120 \mathrm{mmHg}$ de diastólica $)^{55}$.

A las pacientes de alto riesgo trombótico con contraindicación de tromboprofilaxis farmacológica se les debe ofrecer tromboprofilaxis mecánica, preferentemente compresión neumática intermitente ${ }^{54,55}$.

En cuanto a la dosis de HBPM a ser utilizada se recomienda en forma general para pacientes entre 50 y $90 \mathrm{~kg}$ (peso inicio embarazo) enoxaparina $40 \mathrm{mg}$ s.c/día o nadroparina $0.4 \mathrm{~mL}$ s.c/día (3800 UI anti Xa). No requiere monitorización con hemograma ni niveles anti-Xa ${ }^{55}$. En pacientes con insuficiencia renal se sugiere utilizar dosis menores. En situaciones con pesos por debajo de $50 \mathrm{Kg}$ o superior a $90 \mathrm{Kg}$ se requiere ajuste de dosis (tabla 5).

\begin{tabular}{|l|l|l|}
\hline \multicolumn{2}{|c|}{ PESO (KG) } & \multicolumn{1}{c|}{ NADROPARINA } \\
\hline$<50$ & $20 \mathrm{mg} / \mathrm{día}$ & $0.3 \mathrm{ml} /$ día (2.850 UI) \\
\hline $50-90$ & $40 \mathrm{mg} / \mathrm{día}$ & $0.4 \mathrm{ml} /$ día (3800 UI) \\
\hline $91-130$ & $60 \mathrm{mg} / \mathrm{día}$ & $0.6 \mathrm{ml} / \mathrm{día}(5700 \mathrm{UI})$ \\
\hline $131-170$ & $80 \mathrm{mg} / \mathrm{día}$ & $0.8 \mathrm{ml} / \mathrm{día}(7600 \mathrm{UI})$ \\
\hline$>170$ & $\begin{array}{l}0.6 \mathrm{mg} / \mathrm{kg} / \mathrm{día} \\
\text { (en } 102 \text { dosis) }\end{array}$ & \\
\hline $\begin{array}{l}\text { Dosis intermedia } \\
\text { (entre 50-90KG) }\end{array}$ & $40 \mathrm{mg} \mathrm{c} / 12 \mathrm{hs}$ & $0.4 \mathrm{ml}$ (3800 UI) c/12hs \\
\hline
\end{tabular}

En mujeres con indicación de anticoagulación previo al embarazo (por ej: TEV previa asociada a deficiencia de antitrombina o a Síndrome Antifosfolípidico (SAF) o en aquellas con TEV recurrente) se continúa con HBPM a dosis de anticoagulación (enoxaparina $1 \mathrm{mg} / \mathrm{kg}$ cada $12 \mathrm{hs}$ o 1,5mg/kg día o dosis equivalentes de otras) durante todo embarazo y puerperio. Hacia el día 5 a 7 del puerperio se superpone la anticoagulación oral con antivitamina K. En caso de deficiencia de AT con antecedentes de TEV algunos autores recomiendan realizar dosis de anticoagulación y realizar monitorización con anti-Xa para ajustar dosis a niveles entre 0,5 y 1 $\mathrm{UI} / \mathrm{mL}^{55}$.

Para el caso de factores de riesgos transitorios como la hiperémesis gravídica del primer trimestre, el síndrome de hiperestimulación ovárica, entre otros, la tromboprofilaxis se retira cuando la situación que llevó a la indicación se resolvió ${ }^{55}$.

Las mujeres que están recibiendo anticoagulantes orales previo al embarazo, deben ser aconsejadas acerca del riesgo de estos fármacos durante la gestación. Para los anticoagulantes orales directos (ACOD) existe escasa evidencia para hacer recomendaciones actualmente, por lo que se recomienda cambiar a HBPM lo más precozmente posible, con resultado de test de embarazo positivo. En forma similar, en caso de tratamiento con warfarina, se recomienda cambiar a HBPM antes de la semana 6 de gestación por el riesgo de embriopatía ${ }^{59,60}$.

Las pacientes que reciben fármacos antitrombóticos durante el embarazo deben ser educadas acerca del manejo de los mismos al final del embarazo. Se debe sugerir que de presentar genitorragia o iniciar el trabajo de parto no deben administrarse la siguiente dosis de HBPM y deben consultar. El manejo de la anestesia regional y cesárea en pacientes que reciben HBPM se discute en la revisión "Anestesia regional en la paciente embarazada antiagregada o antcoagulada". 


\section{Bibliografía}

1- Morris JM, Algert CS, Roberts CL. Incidence and risk factors for pulmonary embolism in the postpartum period. J. Thromb. Haemost. 2010;8(5):998-1003.

2- Heit JA, Kobbervig CE, James $A H$, et al. Trends in the incidence of venous thromboembolism during pregnancy or postpartum: a 30-year population-based study. Ann. Intern. Med. 2005;143(10):697-706

3- Greer IA. Thrombosis in pregnancy: maternal and fetal issues. Lancet. 1999;353(9160):1258-1265.

4- Marik PE, Plante LA. Venous Thromboembolic Disease and Pregnancy. N. Engl. J. Med. 2008;359(19):2025-2033.

5- Panting-Kemp A, Geller SE, Nguyen T, Simonson L, Nuwayhid B, Castro L. Maternal deaths in an urban perinatal network, 1992-1998. Am. J. Obstet. Gynecol. 2000;183(5):1207-12.

6- Ray JG, Chan WS. Deep vein thrombosis during pregnancy and the puerperium: a meta-analysis of the period of risk and the leg of presentation. Obstet. Gynecol. Surv. 1999;54(4):265-71.

7- Bourjeily G, Paidas M, Khalil H, Rosene-Montella K, Rodger M. Pulmonary embolism in pregnancy. Lancet. 2010;375(9713):500-512.

8- Jacobsen AF, Skjeldestad FE, Sandset PM. Incidence and risk patterns of venous thromboembolism in pregnancy and puerperium?a register-based case-control study. Am. J. Obstet. Gynecol. 2008;198(2):233.e1-233.e7.

9- Pomp ER, Lenselink AM, Rosendaal FR, Doggen CJM. Pregnancy, the postpartum period and prothrombotic defects: risk of venous thrombosis in the MEGA study. J. Thromb. Haemost. 2008;6(4):632-7.

10- Gherman RB, Goodwin TM, Leung B, Byrne JD, Hethumumi R, Montoro M. Incidence, clinical characteristics, and timing of objectively diagnosed venous thromboembolism during pregnancy. Obstet. Gynecol. 1999;94(5 Pt 1):730-4.

11- James AH, Tapson VF, Goldhaber SZ. Thrombosis during pregnancy and the postpartum period. Am. J. Obstet. Gynecol. 2005;193(1):216-219.

12- Larsen TB, Sørensen HT, Gislum M, Johnsen SP. Maternal smoking, obesity, and risk of venous thromboembolism during pregnancy and the puerperium: a population-based nested case-control study. Thromb. Res. 2007;120(4):505-9.

13- Lindqvist P, Dahlbäck B, Mar冈ál K. Thrombotic risk during pregnancy: a population study. Obstet. Gynecol. 1999;94(4):595-9.

14- James AH. Pregnancy-associated thrombosis. Hematol. Am. Soc. Hematol. Educ. Progr. 2009;2009(1):277-85.

15- Battinelli EM, Bauer KA. Thrombophilias in pregnancy. Hematol. Oncol. Clin. North Am. 2011;25(2):32333, viii.

16- Robertson L, Wu O, Langhorne P, Twaddle S, Clark P, Lowe GD, et al. Thrombophilia in pregnancy: a systematic review. Br. J. Haematol. 2006;132(2):171-96.

17- Chan WS, Lee A, Spencer FA, Crowther M, Rodger M, Ramsay T, et al. Predicting deep venous thrombosis in pregnancy: out in \&quot;LEFt\&quot; field? Ann. Intern. Med. 2009;151(2):85-92.

18- Le Moigne E, Genty C, Meunier J, Arnoult AC, Righini M, Bressollette L, et al. Validation of the LEFt score, a newly proposed diagnostic tool for deep vein thrombosis in pregnant women. Thromb. Res. $2014 ; 134(3): 664-7$

19- Royal College of Obstetricians and Gynaecologists. Thromboembolic disease in pregnancy and the puerperium: Acute management. London: RCOG; 2007. (Green-top Guideline No. 28)

20- Bates SM, Greer IA, Middeldorp S, Veenstra DL, Prabulos AM, Vandvik PO. VTE, Thrombophilia, Antithrombotic Therapy, and Pregnancy. Chest. 2012;141(2):e691S-e736S.

21- Raţiu A, Navolan D, Spătariu I, Biriş M, Miculiţă M, Motoc A. Diagnostic value of a negative single color duplex ultrasound in deep vein thrombosis suspicion during pregnancy. Rev. Med. Chir. Soc. Med. Nat. lasi. 114(2):454-6.

22- Chan W-S, Spencer FA, Lee AYY, Chunilal S, Douketis JD, Rodger M, et al. Safety of withholding anticoagulation in pregnant women with suspected deep vein thrombosis following negative serial compression ultrasound and iliac vein imaging. CMAJ. 2013;185(4):E194-200 
23- Ray JG, Vermeulen MJ, Bharatha A, Montanera WJ, Park AL. Association Between MRI Exposure During Pregnancy and Fetal and Childhood Outcomes. JAMA. 2016. Sep 6;316(9):95261. doi: $10.1001 /$ jama.2016.12126.

24- University of California San Francisco; Departament of Radiology and Biomedical Imaging. CT and MR Pregnancy Guidelines: Guidelines for the Use of CT and MRI.

During Pregnancy and Lactation [Internet] UCSF: California; 2021 [cited junio 2021] Available in: https://radiology.ucsf.edu/patient-care/patient-safety/ct-mri-pregnancy\#accordion-pregnancy-and-mriinpatients

25- Brent R, Gorson R. Radiation exposure in pregnancy. Curr. Probl. Radiol. 1972;1-48.

26- National Council on Radiation Protection and Measurements. Medical radiation exposure of pregnant and potentially pregnant women. Maryland: NCRP; 1977 . Report $n^{\circ} 54$.

27- International Commission on Radiological Protection. Pregnancy and Medical Radiation. Ann. ICRP 2000; 30 (1). ICRP Publication No84.

28- Winer-Muram HT, Boone JM, Brown HL, Jennings SG, Mabie WC, Lombardo GT. Pulmonary Embolism in Pregnant Patients: Fetal Radiation Dose with Helical CT. Radiology. 2002;224(2):487-492.

29- Hurwitz LM, Yoshizumi T, Reiman RE, Goodman PC, Paulson EK, Frush DP, et al. Radiation Dose to the Fetus from Body MDCT During Early Gestation. Am. J. Roentgenol. 2006;186(3):871-876.

30- Doshi SK, Negus IS, Oduko JM. Fetal radiation dose from CT pulmonary angiography in late pregnancy: a phantom study. Br. J. Radiol. 2008;81(968):653-658.

31- Greer IA. Thrombosis in pregnancy: updates in diagnosis and management. Hematol. Am. Soc. Hematol. Educ. Progr. 2012;2012:203-7.

32- Che Yaakob CA, Dzarr AA, Ismail AA, Zuky Nik Lah NA, Ho JJ. Anticoagulant therapy for deep vein thrombosis (DVT) in pregnancy. Cochrane Database Syst Rev. 2010 Jun 16;(6):CD007801. doi: 10.1002/14651858.CD007801.

33- Greer IA, Nelson-Piercy C. Low-molecular-weight heparins for thromboprophylaxis and treatment of venous thromboembolism in pregnancy: a systematic review of safety and efficacy. Blood. 2005;106(2):401-407.

34- Royal College of the Obstetricians and Gynaecologists. Thromboembolic Disease in Pregnancy and the Puerperium: Acute Management (Green-top Guideline 37b). London: RCOG; 2015.

35- Tang A-W, Greer I. A systematic review on the use of new anticoagulants in pregnancy. Obstet. Med. 2013;6(2):64-71.

36- Patel JP, Green B, Patel RK, Marsh MS, Davies JG, Arya R. Population pharmacokinetics of enoxaparin during the antenatal period. Circulation. 2013 Sep 24;128(13):1462-9. doi: 10.1161/ CIRCULATIONAHA.113.003198.

37- Voke J, Keidan J, Pavord S, Spencer NH, Hunt BJ; British Society for Haematology Obstetric Haematology Group. The management of antenatal venous thromboembolism in the UK and Ireland: a prospective multicentre observational survey. Br J Haematol. 2007 Nov;139(4):545-58. doi: 10.1111/j.1365-2141.2007.06826.x.

38- Rodie VA, Thomson AJ, Stewart FM, Quinn AJ, Walker ID, Greer IA. Low molecular weight heparin for the treatment of venous thromboembolism in pregnancy: a case series. BJOG. 2002 Sep;109(9):10204. doi: 10.1111/j.1471-0528.2002.01525.x.

39- Andersen AS, Berthelsen JG, Bergholt T. Venous thromboembolism in pregnancy: prophylaxis and treatment with low molecular weight heparin. Acta Obstet. Gynecol. Scand. 2010;89(1):15-21.

40- Greer I, Hunt BJ. Low molecular weight heparin in pregnancy: current issues. Br. J. Haematol. 2005;128(5):593-601.

41- Kamel H, Navi BB, Sriram N, Hovsepian DA, Devereux RB, Vet MS, et al. Risk of a Thrombotic Event after the 6-Week Postpartum Period. N. Engl. J. Med. 2014;370(14):1307-1315.

42- Gándara E, Carrier M, Rodger M. Intermediate doses of low-molecular-weight heparin for the long-term treatment of pregnancy thromboembolism. A systematic review. Thromb. Haemost. 2014;111(03):559561.

43- Kawamata K, Chiba Y, Tanaka R, Higashi M, Nishigami K. Experience of temporary inferior vena cava filters inserted in the perinatal period to prevent pulmonary embolism in pregnant women with deep vein thrombosis. J. Vasc. Surg. 2005;41(4):652-6. 
44- Liu Y, Sun Y, Zhang S, Jin X. Placement of a retrievable inferior vena cava filter for deep venous thrombosis in term pregnancy. J. Vasc. Surg. 2012;55(4):1042-7.

45- Rodger MA, Phillips P, Kahn SR, James AH, Konkle BA; PROSPER Investigators. Low-molecularweight heparin to prevent postpartum venous thromboembolism. A pilot randomised placebocontrolled trial. Thromb Haemost. 2015 Jan;113(1):212-6. doi: 10.1160/TH14-06-0485.

46- Sachdeva A, Dalton M, Amaragiri SV, Lees T. Elastic compression stockings for prevention of deep vein thrombosis. Cochrane Database Syst Rev. 2010 Jul 7;(7):CD001484. doi: 10.1002/14651858. CD001484.

47- James A. The American Congress of Obstetricians and Gynecologists (ACOG) Committee of Practice Bulletins: obstetrics practice bulletin \#138 inherited thrombophilias in pregnancy. Obstet. Gynecol. 2013;122(3):706-717.

48- Marik PE, Plante LA. Venous thromboembolic disease and pregnancy. N Engl J Med. 2008 Nov 6;359(19):2025-33. doi: 10.1056/NEJMra0707993

49- Kourlaba G, Relakis J, Kontodimas S, Holm MV, Maniadakis N. A systematic review and meta-analysis of the epidemiology and burden of venous thromboembolism among pregnant women. Int $\mathrm{J}$ Gynaecol Obstet. 2016 Jan;132(1):4-10. doi: 10.1016/j.ijgo.2015.06.054.

50- Rodger MA, Gris JC, de Vries JIP, Martinelli I, Rey É, Schleussner E, et al. Low-molecular-weight heparin and recurrent placenta-mediated pregnancy complications: a meta-analysis of individual patient data from randomised controlled trials. Lancet. 2016;388(10060):2629-2641.

51- Greer IA, Nelson-Piercy C. Low-molecular-weight heparins for thromboprophylaxis and treatment of venous thromboembolism in pregnancy: a systematic review of safety and efficacy. Blood. 2005;106(2):401-407.

52- Grille S, Vitureira G, Morán R, Retamosa L, Alonso V, Gómez LM, et al. Compliance with the 2009 Royal College of Obstetricians and Gynaecologists guidelines for venous thromboembolic disease prophylaxis in pregnancy and postpartum period in Uruguay. Blood Coagul. Fibrinolysis. 2018;29(3):252-256.

53- Rodger M. Pregnancy and venous thromboembolism: "TIPPS" for risk stratification. Hematology. 2014;2014(1):387-392.

54- Skeith L. Preventing venous thromboembolism during pregnancy and postpartum: crossing the threshold. Hematol. Am. Soc. Hematol. Educ. Progr. 2017;2017(1):160-167.

55- Royal College of the Obstetricians and Gynaecologists. Reducing the Risk of Venous Thromboembolism during Pregnancy and the Puerperium (Green-top Guideline 37b). London: RCOG; 2015.

56- Latino OJ coord. Consenso SOGIBA 2017: Tromboprofilaxis durante el parto y puerperio. Rev Obst Gin Bs As. 2017; 96 (1001).

57- Sénat M-V, Sentilhes L, Battut A, et al. Postpartum practice: guidelines for clinical practice from the French College of Gynaecologists and Obstetricians (CNGOF). Eur. J. Obstet. Gynecol. Reprod. Biol. 2016;202:1-8.

58- Chan WS, Kent NE, Rey E, Corbett T, David M, Douglas MJ, et al; VTE in Pregnancy Guideline Working Group. Venous Thromboembolism and Antithrombotic Therapy in Pregnancy. J. Obstet. Gynaecol. Canada. 2014;36(6):527-553.

59- Cotrufo M, De Feo M, De Santo LS, Romano G, Della Corte A, Renzulli A, et al. Risk of warfarin during pregnancy with mechanical valve prostheses. Obstet. Gynecol. 2002;99(1):35-40.

60- Bates SM, Rajasekhar A, Middeldorp S, McLintock C, Rodger MA, James AH, et al. American Society of Hematology 2018 guidelines for management of venous thromboembolism: Venous thromboembolism in the context of pregnancy. Blood Adv. 2018;2(22):3317-3359.

\section{Aporte de cada autor al trabajo}

Victoria Irigoín: Concepción y diseño del trabajo, recolección de datos, redacción y revisión crítica del manuscrito.

Mariana Lorenzo: Concepción y diseño del trabajo, recolección de datos, redacción y revisión crítica del manuscrito.

Sofía Grille: Concepción y diseño del trabajo, recolección de datos, redacción y revisión crítica del manuscrito.

Valentín González: Concepción y diseño del trabajo, recolección de datos, redacción y revisión crítica del manuscrito. 